\title{
Urban Wiesing (2017) Indikation. Theoretische Grundlagen und Konsequenzen für die ärztliche Praxis
}

\author{
Kohlhammer Verlag, Stuttgart, 163 Seiten, 35,00€, \\ ISBN 978-3-17-033010-8
}

\section{Peter Hucklenbroich}

Online publiziert: 26. Juni 2020

(C) Der/die Autor(en) 2020

Das vorliegende Buch von Urban Wiesing versteht sich als ein Beitrag zur Theorie der Medizin. Anders als frühere Darstellungen des Gebiets, legt Wiesing aber nicht eine medizintheoretische (oder ,epistemologische“) Analyse und Rekonstruktion des gesamten diagnostisch-therapeutischen Procedere der praktischen Medizin vor, sondern konzentriert sich auf den einen Punkt, an dem die normativ-evaluativen Bezugspunkte ins Spiel kommen: die Indikationsstellung und anschließende, mit dem Patienten abgestimmte Entscheidung zur Durchführung einer Intervention.

Die Theorie der Medizin befasst sich traditionell mit der Theorie des praktischen ärztlichen Handelns unter den Bezeichnungen Klinische Methodologie oder Medizinische Entscheidungstheorie (medical decision making). Hier analysiert sie das ärztliche Handeln als eine schritt- und stufenweise Folge von Erkenntnissen, Entscheidungen und Handlungen des Arztes an und mit dem Patienten, die dirigiert wird von den drei Leitfragen (vereinfacht nach Hucklenbroich 1992 und Sox et al. 2013):

1. Reicht die vorliegende Information aus, um therapeutisch handeln zu können, oder müssen weitere Informationen diagnostisch erhoben werden?

2. Welche der insgesamt verfügbaren diagnostischen bzw. therapeutischen Interventionen sind (im vorliegenden Fall) angezeigt?

3. Oder ist gar nichts (mehr) zu tun?

Das sich hieraus typischerweise ergebende schleifenförmige Flussdiagramm ist in der medizintheoretischen Literatur von vielen Autoren dargestellt worden und hat auch zur Vorlage für die Konstruktion entscheidungsunterstützender Systeme mithilfe von Computern und Künstlicher Intelligenz gedient (z. B. Hucklenbroich

Prof. Dr. med. Dr. phil. P. Hucklenbroich $(\bowtie)$

Münster, Deutschland

E-Mail: Peter.Hucklenbroich@ukmuenster.de 
und Toelllner 1993). Es existieren mehrere Standardwerke in englischer Sprache speziell zum Medical Decision Making, während die deutschsprachige Literatur die Klinische Methodologie meist im größeren Zusammenhang der Medizintheorie insgesamt behandelt (z. B. Mannebach 1997; Gross und Löffler 1998).

Der Entscheidungsfluss in der Klinischen Methodologie dient dazu, das allgemeine medizinische Wissen über Krankheiten und Behandlungsmethoden für den jeweiligen Einzelfall optimal zu nutzen. An zwei Stellen wird hier neben dem theoretischen Wissen auf Normen und Werte zurückgegriffen: Zur Beantwortung der Leitfrage 2 - was ist angezeigt? - ist zu berücksichtigen, welche generellen Ziele die Medizin verfolgt (,goals of medicine“) und welche im konkreten Fall erreichbar sind, und bei der endgültigen Auswahl und Durchführung einer Intervention sind die Werte und Präferenzen des konkreten Patienten zu berücksichtigen. Dagegen ist die Entscheidung, welche generellen und individuellen Informationen benötigt werden, um überhaupt erfolgreich behandeln zu können, eine kognitive, nicht-normative Frage.

Im vorliegenden Buch gibt Wiesing Definitionen für die Begriffe der Indikationsstellung, der Indikationsregel und des Indikationsgebiets an, und diskutiert im Hauptteil, in Auseinandersetzung mit der aktuellen medizinethischen und medizinrechtlichen Literatur, ausgesprochen kleinteilig und redundant in 50 Unterkapiteln, welche Vorzüge diese seine Definitionen gegenüber anderen Auffassungen in der Literatur (seiner Meinung nach) haben.

Eingebettet in diesen Hauptteil skizziert Wiesing einige Thesen, mit denen er sich in der medizintheoretischen Diskussion zu positionieren sucht, ohne jedoch auf diese Diskussion ausführlicher einzugehen. Dies sind die den Rezensenten am wenigsten überzeugenden und kaum informativen Teile des Buches. Die drei wichtigsten Thesen lauten:

1. Die Medizin besteht aus zwei Bereichen, die beide Wissenschaften sind. Eine von Wissenschaft unterscheidbare ärztliche Praxis gibt es nicht.

2. Neben der krankheitsbezogenen ärztlichen Indikation sollte es eine Indikation ohne Krankheitsbezug geben. In diesem Bereich, nicht im krankheitsbezogenen Vorgehen, liegt voraussichtlich die Zukunft der Medizin.

3. Die im Buch vorgestellten Definitionen und Begriffe von Indikation und Indikationsregeln sind hier erstmalig von Wiesing entwickelt worden. Es gibt keine medizintheoretischen Arbeiten in der jüngeren und aktuellen Literatur, die sich mit diesen Begriffen befassen.

Allen drei Thesen muss widersprochen werden, ohne dass in dieser Rezension die eigentlich erforderliche Sachdiskussion nachgeholt werden könnte:

1. Dass das ärztliche Handeln an und mit Patienten, außerhalb von klinischen Studien, als Wissenschaft zu gelten habe und es daher eine ärztliche Praxis, die nicht zugleich Wissenschaft wäre, gar nicht gibt, ist eine extreme und in der Wissenschaftstheorie kaum vertretene, kontroverse Position. Der von Autoren wie Richard Koch, Wolfgang Wieland oder Hans-Georg Gadamer ins Spiel gebrachte Begriff einer ,praktischen Wissenschaft Medizin“ ist keineswegs, wie manchmal behauptet wird, durch die aristotelische Wissenschaftslehre gedeckt. Die Gegen- 
position besagt, dass klinisch-ärztliches (Be-)Handeln als eine verwissenschaftlichte und wissenschaftsorientierte Praxis aufzufassen ist, analog zu dieser Unterscheidung in den Erfahrungswissenschaften Physik, Chemie und Biologie. Da Wiesing es unterlässt, die Gegenposition auch nur zu erwähnen, geschweige denn die Argumente Pro und Contra zu referieren, ist dieser Teil des Buches wissenschaftlich gesehen mindestens irrelevant.

2. Wiesings Konstrukt einer „Indikation ohne Krankheitsbezug“ erscheint wenig durchdacht: Auf welches Wissen soll sich ein Arzt hinsichtlich der Wirksamkeit und Prognose beziehen, wenn er etwa die Anwendung medizinischer Interventionen für sportliche, militärische oder biotechnische Zwecke beurteilen soll, soweit es hierbei ja gerade nicht um medizinisches Krankheitswissen gehen soll? Der Arzt müsste dann ja wohl Experte für sportliche Tätigkeiten, militärische Strategien und biotechnische Verfahren werden - und wäre insofern gerade nicht mehr Arzt.

3. Den schwersten Fehler stellt die dritte These dar. Die Behauptung, dass die vorgestellten Definitionen erstmalig in diesem Buch entwickelt seien, irritiert angesichts dessen, dass die medizintheoretischen Grundlagen und begrifflichen Klärungen bezüglich Indikation und Indikationsregeln sich sämtlich in 15 Publikationen der letzten Jahre von der Hand dieses Rezensenten finden (z. B. Hucklenbroich 1992, 2013, 2016), von denen keine einzige im Literaturverzeichnis des rezensierten Buches genannt wird. Im günstigsten Fall muss man Wiesing Unkenntnis des wissenschaftlichen Diskussionsstandes vorwerfen. Auf jeden Fall ist seine Kritik an der angeblichen Lücke in der medizintheoretischen Literatur und sein Originalitätsanspruch unbegründet.

Funding Open Access funding provided by Projekt DEAL.

Open Access Dieser Artikel wird unter der Creative Commons Namensnennung 4.0 International Lizenz veröffentlicht, welche die Nutzung, Vervielfältigung, Bearbeitung, Verbreitung und Wiedergabe in jeglichem Medium und Format erlaubt, sofern Sie den/die ursprünglichen Autor(en) und die Quelle ordnungsgemäß nennen, einen Link zur Creative Commons Lizenz beifügen und angeben, ob Änderungen vorgenommen wurden.

Die in diesem Artikel enthaltenen Bilder und sonstiges Drittmaterial unterliegen ebenfalls der genannten Creative Commons Lizenz, sofern sich aus der Abbildungslegende nichts anderes ergibt. Sofern das betreffende Material nicht unter der genannten Creative Commons Lizenz steht und die betreffende Handlung nicht nach gesetzlichen Vorschriften erlaubt ist, ist für die oben aufgeführten Weiterverwendungen des Materials die Einwilligung des jeweiligen Rechteinhabers einzuholen.

Weitere Details zur Lizenz entnehmen Sie bitte der Lizenzinformation auf http://creativecommons.org/ licenses/by/4.0/deed.de.

\section{Literatur}

Gross R, Löffler M (1998) Prinzipien der Medizin. Eine Übersicht ihrer Grundlagen und Methoden. Springer, Berlin

Hucklenbroich P, Toellner R (Hrsg) (1993) Künstliche Intelligenz in der Medizin. G. Fischer, Stuttgart

Hucklenbroich P (1992) Wissenschaftstheorie als Theorie der Medizin: Themen und Probleme. In: Deppert $\mathrm{H}$ et al (Hrsg) Wissenschaftstheorien in der Medizin. de Gruyter, Berlin/New York, S 65-93 
Hucklenbroich P (2013) Die wissenschaftstheoretische Struktur der medizinischen Krankheitslehre. In: Hucklenbroich P, Buyx AM (Hrsg) Wissenschaftstheoretische Aspekte des Krankheitsbegriffs. Mentis, Münster, S 13-83

Hucklenbroich P (2016) Die Normativität des Krankheitsbegriffs: Zur Genese und Geltung von Kriterien der Krankhaftigkeit. Anal Krit 38(2):459-496

Mannebach H (1997) Die Struktur des ärztlichen Denkens und Handelns. Chapman \& Hall, London

Sox HC, Higgins MC, Owens DK (2013) Medical decision making. Wiley-Blackwell, Chichester 